RU-98-02

\title{
Remark on lattice BRST invariance.
}

\author{
Herbert Neuberger \\ Department of Physics and Astronomy \\ Rutgers University, Piscataway, NJ 08855-0849
}

\begin{abstract}
A recently claimed resolution to the lattice Gribov problem in the context of chiral lattice gauge theories is examined. Unfortunately, I find that the old problem remains.
\end{abstract}


Within the BRST framework, perturbation theory indicates that the trivial Gaussian fixed point associated with an asymptotically free chiral four dimensional gauge theory has only one (marginally) unstable direction associated with the gauge coupling. If the requirement of BRST invariance is relaxed, one has a larger, but finite, number of unstable directions, and the approach to the desired continuum limit is conditional on fine tuning to eliminate the effects of the extra unstable directions. The fine tuning can be specified imposing additional identities, one for each extra unstable direction. One chooses identities that would hold if BRST invariance were exact. The RG then indicates that full BRST invariance will be restored in the continuum limit, and that a self-consistent, potentially strongly coupled, chiral gauge theory exists also outside perturbation theory. This is my understanding of the essence of the "Rome proposal" [1]. About a decade ago, when lattice chiral gauge theory still amounted mostly to an industry of failures, the Rome proposal triggered a process of restoring common sense to this subfield.

The one nagging suspicion about the Rome proposal that I have is the lack of certainty surrounding BRST invariance at the non-perturbative level in simpler, non-chiral, lattice gauge theory. This, in addition to some lack of clarity regarding global anomalies [2], indicates that even in principle, the Rome proposal may need a (hopefully small) upgrade or "bug-fix" [3]. In the rest of this note I shall have nothing more to say about global anomalies and shall focus only on non-perturbative BRST invariance.

In the late seventies to mid eighties, after some confusion having to do with Gribov copies, it became clear that as long as all solutions to the gauge fixing constraints were included together with the associated full Faddeev-Popov determinants (with their signs) no formal problems of principle were evident [4]. Unfortunately, using a direct transplant to the lattice [5] of the continuum BRST operation (interpreted in the language of Lie Algebra Cohomology with the addition of a Koszul-Tate resolution), it was found that all BRST invariant observables have the form $\frac{0}{0}$ [6]. This would imply, in particular, that applying the Rome approach to QCD for example, produces an ill defined theory. Moreover, one cannot imagine a lattice RG transformation that produces a fixed point and a one dimensional renormalization group trajectory emanating from it in the one marginal direction available, along which all actions are BRST invariant, corresponding to an anomaly free, asymptotically free, chiral gauge theory. Note that the BRST transformation is always a local operator, since it simply encodes local gauge invariance. While these observations do not constitute proof that the Rome proposal has a defect, it seems to me that they do provide grounds to ask for an unequivocal resolution.

In a stream of recent publications [7] it has been repeatedly claimed that the above problem can be avoided by choosing a special gauge fixing term in the Lagrangian. The 
term depends only on the gauge fields, has an absolute minimum when all lattice parallel transporters equal unity, and is naturally written as the sum of two terms rather than the square of a single local factor. Since most of this work is directed to the $U(1)$ case, the issue of asymptotic freedom being somewhat divorced from the problems encountered with lattice chirality, I shall restrict myself to the $U(1)$ case below.

As far as I can see, the gauge fixing employed in [7] fits into the lattice BRST framework of $[5,6]$ exactly as well as the original proposal [1]. The somewhat different structure of the gauge fixing term is irrelevant to the lattice BRST problem I am addressing. For example, even with the gauge fixing term of [7], BRST invariant lattice QED will still produce $\frac{0}{0}$ for the expectation value of any BRST invariant observable. The unfortunate conclusion is that no progress of principle beyond the Rome proposal has been made in [7]. I am leaving open the possibility that I am making a mistake (for which I wish to apologize in advance) because the argument below is so simple that it is hard to imagine it being overlooked in all the papers in [7].

The basic issue boils down to this: Consider the full action with all Grassmann variables set to zero. This action splits additively into a gauge invariant term and a gauge fixing term. The question now is whether it is possible, by the addition of terms involving only BRST ghosts, to restore full BRST invariance. The answer is positive both in the case of [1] and in the case of [7]. When the non-ghost fermion fields are turned on BRST invariance is lost both in [1] and in [7]; but this is besides the point I am making here. The easiest way to check a particular case is to go through the formal steps one would take to implement the Faddeev Popov trick on the lattice. While the steps contain an error when viewed as identities they do produce a path integral that could be taken as the definition of a BRST invariant, nonperturbatively defined, field theory. As explained in [6], if the steps were mathematically correct we would, of course, end up with a logical contradiction to the result of $[6]$, since a perfectly reasonable lattice gauge invariant theory would be proven equivalent to a nonsensical one. In the next few lines I sketch these formal steps for the gauge fixing term of [7]. The conclusion would be that the gauge fixing term of [7] is not special at all, and can be incorporated in standard lattice BRST, as used in [1] and in $[5,6]$.

The gauge fixing term of [7], appearing with a minus sign in the exponent in the standard formula for the partition function $(\alpha>0)$, can be written as

$$
\frac{1}{2 \alpha} \sum_{x} S(U ; x),
$$

where $x$ denotes lattice sites, and $U$ the collection of $U(1)$ link variables $U_{\mu}(y) . S(U, x)$ is local in that it depends only on $U_{\mu}(y)$ with $y$ near $x$. For all $U, S(U, x) \geq 0$ and $S(\mathbf{1}, x)=0$ 
for all $x$. Since the $U_{\mu}(y)$ are compact there exists a number $M>1$, such that for all $U$ and $x, S(U, x) \leq M-1$. Let $f(U, x)$ be a gauge fixing function defined as follows:

$$
f(U, x)=\sqrt{M+S(U, x)}
$$

In (2) I chose the positive branch of the square root. Clearly, $f(U, x)$ is a uniformly converging series in $S(U, x)$. Introducing a product of delta functions, $\prod_{x} \delta(b(x)-f(U, x))$, into the path integral of a gauge invariant model, adding the Faddeev-Popov determinant factor, and averaging over $b(x)$ with a Gaussian weight $\exp \left[-\frac{1}{2 \alpha} \sum_{x} b^{2}(x)\right]$, produces the bosonic gauge fixing part of equation 1 up to an irrelevant multiplicative constant

$\exp \left[-\frac{1}{2 \alpha} \sum_{x} M\right]$. The result of [6] directly applies and leads to the $\frac{0}{0}$ problem. For this case the ghosts enter only bilinearly so the alternative explanation offered in [6] also applies: the number of solutions to the gauge fixing equations is even (including 0) generically. On the trivial orbit there is a single solution because it is non-generic, being a minimum. However, almost on all orbits in the vicinity of the trivial orbit there will be several solutions making contributions that sum up to zero.

\section{Acknowledgments.}

This research was supported in part by the DOE under grant \# DE-FG05-96ER40559. I am indebted to M. Testa who anticipated the result of this note in a question he posed to me during the last lattice conference; unfortunately I was too dense to see immediately then that he was right. I also wish to thank Y. Kikukawa for a discussion. In addition I wish to thank J. Smit for alerting me to possible misunderstandings that a somewhat more tersely worded earlier version of this note could have triggered.

\section{References.}

[1] A. Borelli, L. Maiani, G.-C. Rossi, R. Sisto and M. Testa, Nucl. Phys. B333 (1990) 335; L. Maiani, G.-C. Rossi and M. Testa, Phys. Lett. 292B (1992) 397.

[2] E. Witten, Phys. Lett. B 117 (1982) 324.

[3] H. Neuberger, Nucl. Phys. B. (Proc. Suppl.) 17 (1990) 17.

[4] P. Hirschfeld, Nucl. Phys. B 157 (1979) 37; B. Sharpe, J. Math Phys. 25 (1984) 3324.

[5] H. Neuberger, Phys. Lett. B 175 (1986) 69.

[6] H. Neuberger, Phys. Lett. B 183 (1987) 337.

[7] Y. Shamir, Phys. Rev. D57 (1998) 132; M. Golterman and Y. Shamir, Phys. Lett. 399B (1997) 148; M. Bock, M. Golterman and Y. Shamir, hep-lat/9709154, heplat/9708019, hep-lat/9709113, hep-lat/9709115, hep-lat/9801018. 